\title{
Liposarcoma: Atypical Spread with Intraperitoneal and Vertebral Metastasis in a Young Female
}

\author{
'Department of Obstetrics and Gynecology, Liv Hospital, İstanbul-Turkey \\ ${ }^{2}$ Department of General Surgery, Liv Hospital, İstanbul-Turkey \\ ${ }^{3}$ Department of Obstetrics and Gynecology, Bezmialem Vakif University, İstanbul-Turkey
}

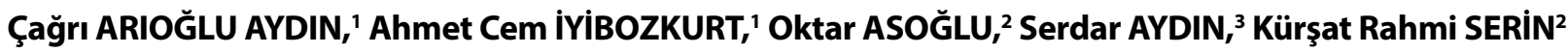

\section{Introduction}

Liposarcomas are among the most frequently encountered soft-tissue malignant tumors. Lesions arise from lipoblasts and occur in many tissues, including fat, muscle, bone, retroperitoneum, and viscera. Tumors vary considerably in presentation, with some classified as high-grade and others as well-differentiated. The World Health Organization currently classifies the tumors into 4 subtypes: i) atypical, well-differentiated, and probably benign, as metastasis is rare, although lesions may locally recur and cause destruction; ii) myxoid/round cell and locally aggressive, with the potential to metastasize; iii) dedifferentiated, very aggressive, and highly malignant; iv) pleomorphic, malignant. [1]

All forms of liposarcomas clinically appear as mass- es, principally located in the extremity soft tissues, muscles, bones, and retroperitoneum.[2] They are less commonly found in other sites. Described in the present report is the case of a 25-year-old female with myxoid liposarcoma, and with huge intraperitoneal mass near the sigmoid colon, uterus, and bilateral adnexa that spread to the vertebra, intraperitoneum, axilla, liver, and abdominal wall, who was treated with neoadjuvant chemoradiotherapy and radical excisions, including the uterus and ovaries.

\section{Case Report}

A young woman with complaint of right thigh pain was admitted to the clinic. Tru-cut biopsy revealed well- 
differentiated myxoid and round cell liposarcoma. After chemotherapy and radiotherapy, the mass was removed with wide local excision of the lesion from the right thigh. Pathology was well-differentiated myxoid and round cell liposarcoma, and surgical margins were negative. After 11 months, a vertebral metastasis at the 11th thoracic vertebra was determined on thoracic computed tomography. Neo-adjuvant chemoradiotherapy was performed, the corpus of the 11th thoracic vertebra was excised, and an implant was placed. Six months later, abdominal-pelvic magnetic resonance imaging (MRI) demonstrated a giant pelvic mass throughout the entire pelvis. The mass was approximately $13 \times 12 \mathrm{~cm}$, behind the uterus, with multiple metastasis in the 2,5 , and 8 segments of the liver. Hysterectomy, lower anterior resection of the sigmoid colon, removal of $8 \mathrm{~cm}$ of jejunum, and side-to-side intestinal anastomosis were performed (Figure 1). Liver metastasis were removed with the aid of intraoperative ultrasonography (Figure 2). Histological review was tumor lesion with high-grade, poorly differentiated myxoid and round cell liposarcoma.

Four months after surgery, masses in the left abdominal wall and left axilla were present. Whole-body diffusion MRI revealed a mass in the left abdominal wall behind the rectus muscle, 2 masses in the left axillary area, and a $3-\mathrm{cm}$ diameter mass near the pancreas. In addition, certain changes in the right tibia corticomedullary section, similar to metastasis, were detected on contrast MRI. A mass behind the pancreas and under the left renal artery, the left rectus muscle, and the masses near the axillary nerve, vein, and artery were removed with capsule. Finally, biopsy was obtained from the right tibia. Pathology reported that the tumor behind the pancreas was a low-grade myxoid liposarcoma without round cell component, while the tumor from the abdominal wall was a myxoid liposarcoma. The axillary tumor was myxoid and round cell liposarcoma, and the axillary lymph nodes were reactive. Tibia biopsy was reported as intraosseous lipoma. The patient has been free of complications and metastasis for 12 months.

\section{Discussion}

Described in the present report is the concomitance of intraperitoneal and vertebral bone involvement with myxoid liposarcoma in a young female, with different presentations. Myxoid liposarcoma is a clinicopathologically and genetically distinct type, characterized by common occurrence in young adults, location in the thigh, and presence of $t(12 ; 16)$ translocation. [2,3] Undifferentiated and pleomorphic types are neoplasms with high grade of malignancy, accompanied by remarkable biological aggressiveness with metastatic potential. Well-differentiated and myxoid/round cell forms are tumors with low grade of malignancy, associated with more favorable prognosis.[4]

By definition, multicentric liposarcoma is a lesion that develops in any typical location of primary liposarcoma, such as the thigh, retroperitoneum, arm, or pleura, without metastasis to conventional areas such as the lung, and having a differentiated histologic type. [5] Diagnosis of this uncommon condition is not wellestablished, with differentiation between multiple primary lesions and metastasis of a single tumor being the main problem. One point that can be used to define multicentric tumor is the presence of tumors in areas where metastasis does not usually occur. The most common sites of metastasis are the lung and retroperitoneum. In the present patient, atypical location of tumors supported diagnosis of multicentricity. Cytogenetic techniques can be useful when recognizing or ruling out a colonial relationship between 2 lesions, but unfortunately could not be used to do so in the pres-

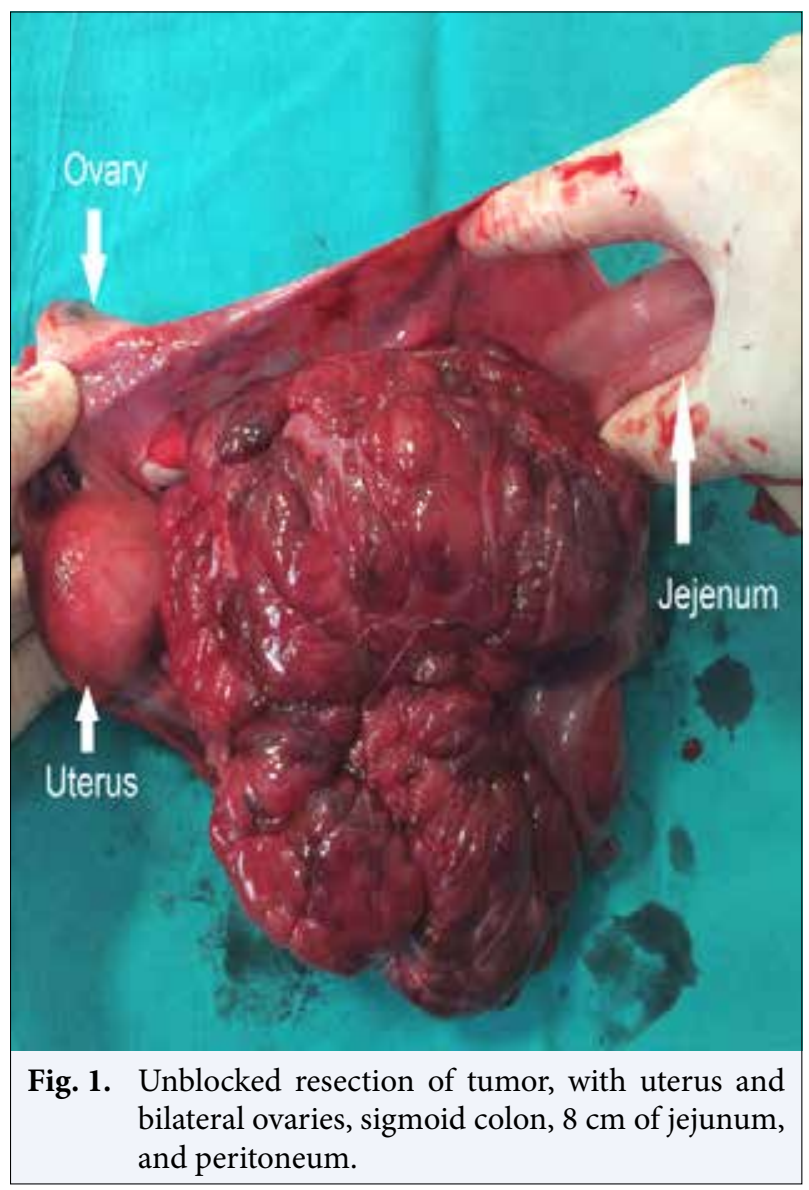




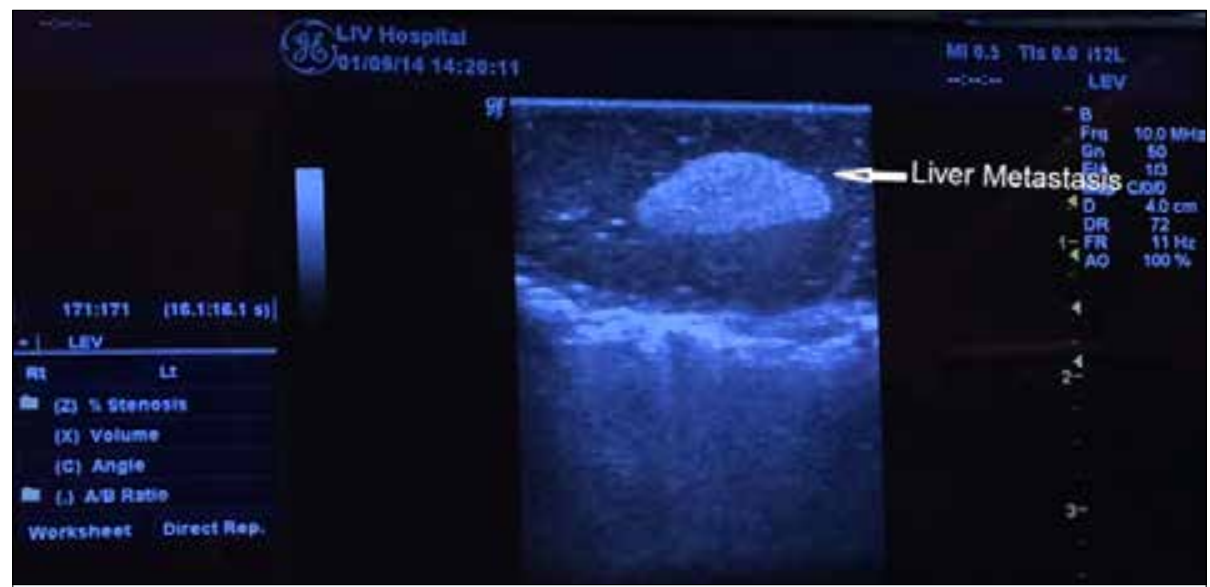

Fig. 2. Intraoperative view of liver metastasis.

ent case. Myxoid and round cell liposarcoma constitute a spectrum of the same condition and various histological forms of liposarcoma. Advances in cytogenetic techniques have made identification of the characteristic markers of liposarcoma possible, $t(12 ; 16)$ (q13;p11) translocation in particular, which is seen in the myxoid and round cell subtypes.[6] Pure myxoid liposarcoma is the second most common type, comprising $30 \%$ of cases.[2,3] Round cell liposarcoma is defined as detection of $>5 \%$ round cell component in myxoid liposarcoma, and constitutes $15 \%$ of all liposarcomas. [2] The subtypes share the same chromosomal translocation, $t(12 ; 16)(q 13 ; p 11)$, which leads to the fusion of the CHOP gene on chromosome 12 with the TLS gene on chromosome 16 and the generation of the TLSCHOP fusion gene.[7] Therefore, these subtypes are accepted as 2 different entities and biological diversity of the same tumor.[2,3] In the present case, the index tumor of the thigh was well-differentiated myxoid and round cell liposarcoma. The vertebral, intraperitoneal, and liver metastases were high-grade, poorly differentiated myxoid and round cell liposarcoma. Metastasis of the abdominal wall and intraperitoneal tumor near the pancreas did not have a round cell component. Axillary tumor had $<5 \%$ round cells in the present case.

Well-differentiated liposarcoma more commonly occurs in patients between 50 and 70 years of age, and has practically no risk of distant spread. In contrast, myxoid liposarcoma often occurs in patients aged 25-45 years and has high incidence of metastases. $[1,8]$ In the present case, the patient was 25 years old at the 2 nd operation and 24 years old at 1st diagnosis, making her the youngest reported patient with adult liposarcoma with atypical spread.

Myxoid liposarcoma represents low-grade sarcoma, while round cell liposarcoma suggests high-grade liposarcoma.[3] Myxoid and round cell liposarcoma are sensitive to radiotherapy and chemotherapy, in contrast to other liposarcoma types.[2] Radical excision of the tumor offers the possibility of longer survival and a disease-free interval. $[9,10]$ In all patients reviewed in the literature, surgical excision was the first line of treatment for intraperitoneal liposarcoma. The value of adjuvant chemotherapy has not been established. Postoperative radiotherapy of the whole tumor bed is not feasible at a tolerable toxicity.

Demonstrated by the present case is the possibility of diffuse vertebral, intraperitoneal, axillary, and bone involvement of myxoid liposarcoma in a young female with an initial well-differentiated myxoid/round cell tumor. This uncommon pattern of spread should certainly be taken into account when staging patients and determining prognosis. The value of MRI in the detection of these metastases is also shown, as is the need for close follow-up.

\section{Acknowledgment}

The authors would like to thank the contributors to this report.

\section{Disclosure Statement}

The authors declare no conflicts of interest.

\section{References}

1. Murray PM. Soft tissue sarcoma of the upper extremity. Hand Clin 2004;20(3):325-33.

2. Fiore M, Grosso F, Lo Vullo S, Pennacchioli E, Stacchi- 
otti S, Ferrari A, et al. Myxoid/round cell and pleomorphic liposarcomas: prognostic factors and survival in a series of patients treated at a single institution. Cancer 2007;109(12):2522-31.

3. ten Heuvel SE, Hoekstra HJ, van Ginkel RJ, Bastiaannet E, Suurmeijer AJ. Clinicopathologic prognostic factors in myxoid liposarcoma: a retrospective study of 49 patients with long-term follow-up. Ann Surg Oncol 2007;14(1):222-9.

4. Crago AM, Singer S. Clinical and molecular approaches to well differentiated and dedifferentiated liposarcoma. Curr Opin Oncol 2011;23(4):373,8.

5. Sato H, Minei S, Sugimoto S, Kishimoto Y, Yoshida T, Takimoto Y. Multicentric liposarcoma. Int J Urol 2004;11(12):1133-5.

6. Knight JC, Renwick PJ, Dal Cin P, Van den Berghe H, Fletcher CD. Translocation $\mathrm{t}(12 ; 16)(\mathrm{q} 13 ; \mathrm{p} 11)$ in myx- oid liposarcoma and round cell liposarcoma: molecular and cytogenetic analysis. Cancer Res 1995;55(1):24-7.

7. Dalal KM, Antonescu CR, Singer S. Diagnosis and management of lipomatous tumors. J Surg Oncol 2008;97:298-313.

8. Singer S, Antonescu CR, Riedel E, Brennan MF. Histologic subtype and margin of resection predict pattern of recurrence and survival for retroperitoneal liposarcoma. Ann Surg 2003;238(3):358-71.

9. Karaman A, Kabalar ME, Ozcan O, Koca T, Binici DN. Intraperitoneal dedifferentiated liposarcoma: a case report. World J Gastroenterol 2008;14(38):5927-9.

10. Hasegawa T, Seki K, Hasegawa F, Matsuno Y, Shimodo T, Hirose T, et al. Dedifferentiated liposarcoma of retroperitoneum and mesentery: varied growth patterns and histological grades--a clinicopathologic study of 32 cases. Hum Pathol 2000;31(6):717-27. 